\title{
Variations
}

Variations

Revue internationale de théorie critique

$20 \mid 2017$

Expériences oppositionnelles

\section{Résistances en ligne : mobilisation, émotion, identité}

Fabien Granjon

\section{(2) OpenEdition}

1 Journals

Édition électronique

URL : http://journals.openedition.org/variations/819

DOI : 10.4000/variations.819

ISSN : 1968-3960

Éditeur

Les amis de Variations

Référence électronique

Fabien Granjon, «Résistances en ligne : mobilisation, émotion, identité », Variations [En ligne], 20 |

2017, mis en ligne le 25 avril 2017, consulté le 25 février 2021. URL : http://journals.openedition.org/ variations/819; DOI : https://doi.org/10.4000/variations.819

Ce document a été généré automatiquement le 25 février 2021.

Les ami•e•s de Variations 


\title{
Résistances en ligne : mobilisation, émotion, identité
}

\author{
Fabien Granjon
}

1 La conflictualité sociale s'appuie sur des « armes matérielles » au nombre desquelles les technologies d'information et de communication (TIC) ont très souvent joué un rôle central ${ }^{1}$. Les big media écrits et audiovisuels (presse, radio et télévision), tout comme les small media nettement plus mobilisables (« ronéo», cassettes audio, vidéo, etc.) font partie des technologies des mouvements sociaux. L'action collective protestataire s'actualise en effet dans des formes d'agir pour le moins variées dont certaines sont assurément en lien avec ces médiations (ce par quoi il faut passer) médiatiques et technologiques qui organisent matériellement la diffusion, la mise en visibilité/ publicité et la discussion de productions symboliques (informations, savoirs, idéologies) prenant part à la conflictualité sociale. Les politiques $d u$ conflit ${ }^{2}$ ont, tout comme la plupart des activités sociales contemporaines, notamment politiques, partie liée avec une variété de supports technologiques qui, aujourd'hui, relèvent assez largement de l'informatique connectée. Celle-ci permet de rassembler plusieurs formes de production médiatique (écrits, sons, images fixes et animées), d'en faciliter la production et l'articulation (intertextualité, interdiscursivité) et de les coupler aisément à des espaces de commentaires, de débats et de sociabilité conduisant potentiellement à l'édification d'espaces publics oppositionnels ${ }^{3}$. Le " réseau des réseaux " est devenu un outil essentiel pour un nombre croissant de mouvements sociaux qui en développent des usages variés, afin d'ouvrir de nouveaux modes de participation à l'action collective, d'élargir le spectre des participants aux dynamiques protestataires, ou encore de faciliter la tenue de mobilisations, parfois de grande ampleur et de haute intensité. Internet et la téléphonie mobile sont ainsi largement mobilisés durant les séquences de conflit ouvert, mais les technologies numériques d'information et de communication (TNIC) font également partie du répertoire des instruments nécessaires à l'entretien des logiques contestataires durant les périodes ordinaires de basse ou moyenne intensité, notamment s'agissant des capacités de mobilisation. Précisément, dans le cadre de cet article, nous souhaitons aborder cet aspect spécifique de la 
matérialité numérique de la conflictualité sociale contemporaine qui tient aux nouvelles modalités de mobilisation de l'action.

\section{De nouvelles communautés pertinentes pour l'action}

2 À la fin des années 1990, les premières enquêtes de terrain menées sur les usages militants de l'Internet ${ }^{4}$ montraient que la probabilité qu'un individu s'engage, suite à la réception d'un appel à mobilisation lancé sur une liste de diffusion restait largement indexée à sa motivation à participer. La participation était d'autant plus forte qu'elle sollicitait des individus ayant le sentiment d'appartenir à une communauté immédiatement concernée par l'objet de la mobilisation et donc ayant, a priori, une plus grande volonté de participer ${ }^{5}$. Aussi, les thèses d'Anthony Oberschall ${ }^{6}$, pour qui la mobilisation pour l'action consiste plutôt à recruter des groupes d'acteurs déjà organisés et actifs, et non à solliciter des individus plus ou moins isolés semblaient également se vérifier s'agissant de l'engagement sur réseaux. L'Internet ne semblait pouvoir jouer un rôle de renforcement des mobilisations in praesentia qu'auprès des individus parmi les plus impliqués dans des activités militantes de terrain. Pour autant, pouvaient se constater, en certaines occasions, des formes d'élargissement de l'engagement vers des personnes qui, par exemple, n'étaient pas nécessairement membres d'une organisation militante. Si les communautés pertinentes pour l'action ${ }^{7}$ restaient donc indexées à un principe d'intégration préalable des sujets agissants à une structure contestataire, il était néanmoins possible d'observer la constitution de fragiles collectivités concrètes de référence tenant davantage à la valorisation d'un "Nous" naissant du partage de services en ligne (pour l'essentiel forums et listes de diffusion à l'époque). Par ailleurs, les formes empiriques de protestation collective relevant de l'hacktivisme étaient encore peu développées, mais elles composaient un faisceau de signaux de moins en moins faibles qui laissaient à penser qu'allaient, à l'avenir, se développer des collectifs erratiques susceptibles de se transformer en communautés d'action à partir, précisément, des expériences que ceux-ci tirent de leurs usages de l'informatique connectée.

3 Quelques années plus tard, émerge notamment un ensemble de collectifs (Anonymous, WikiLeaks, etc.) qui se fonde largement sur l'accrétion de contributeurs qui se constituent en collectifs, nettement moins par l'appartenance ou l'affiliation, mais davantage par la volonté commune et révisable à tout moment de mener une action pour laquelle Internet devient une condition de possibilité essentielle de leur mise en capacité. Clay Shirky ${ }^{8}$ relate ainsi l'improbable mobilisation de jeunes Sud-Coréen(ne)s contre la réouverture des importations de viande bovine états-unienne. Celle-ci n'aurait pas été conduite à l'initiative d'organisations associatives ou politiques, mais aurait été lancée par de jeunes fans d'un boys band qui, fréquentant le forum du groupe, auraient, à un moment donné, polarisé leurs discussions « légères » sur ce thème plus politique et lancé l'idée d'une protestation publique qui aurait finalement abouti. Si, en l'occurrence, la mobilisation en ligne s'est traduite par une manifestation de rue, il est des actions qui se maintiennent dans l'espace numérique. Qualifiées par Romain Badouard de «mobilisations de clavier ", elles se fondent sur la cumulation « d'actions individuelles dans le cadre d'une activité dont l'objectif attendu est souhaité par l'ensemble des internautes qui y prennent part, dans la mesure où il répond à un intérêt commun, ou du moins à des intérêts partagés ${ }^{9}$ ». Attachés à la figure du réseau 
qui n'agrège pas, mais réticule des nœuds, ces collectifs plus ou moins durables réinterrogent la place du sujet dans l'élaboration d'un "Nous ", via le modèle de la connexion dont ils font la forme privilégiée de la constitution communautaire. À l'action tactique « ordinaire » décidée en amont par un collectif qui mobilise à cet effet, s'ajoutent d'autres formes de dialectique Je/Nous: coordination conjoncturelle d'une action, légitimation et reconnaissance des actions individuelles par le collectif, etc.

Les réseaux télématiques tendent à devenir des contextes communs de socialisation et il n'est pas incongru de penser qu'ils peuvent se trouver à l'origine de dispositions (manières d'agir, de penser, etc.) qui, sans être pleinement nouvelles, s'hybrident à une diversité toujours plus grande de contextes sociaux en ligne et hors ligne, et reconfigurent ainsi certaines pratiques sociales au nombre desquelles on compte donc la création de collectifs et de minorités actives davantage tournés vers l'action directe. Les "mouvements de crise" ou certaines actions de type "Take the square", menées lors des «Printemps» arabes (Tunisie, Égypte), d'érable (Québec) ou en Turquie (occupation de la place Taksim et du parc Gezi d'Istanbul), empruntent très certainement au réseau et à son parangon Internet ; pas seulement comme technologies d'information et de communication, mais également comme technologies intellectuelles susceptible d'organiser une structure oppositionnelle labile dont la principale force tiendrait à ce qu'elle est une structure, expression directe de la communauté. Quand un candidat du Parti pirate français déclare : "Nous sommes prêts à travailler avec n'importe quelle formation politique, qu'elle soit de droite ou de gauche, peu importe", sans doute définit-il moins une improbable orientation politique «neutre » qu'il n'exprime cette disposition pragmatique à faire communauté autour d'objectifs qui se définissent dans et par l'action, c'est-à-dire sans préjuger des orientations préalables des personnes prenant part à cette action et sur laquelle, en une situation donnée, ils s'entendent précisément pour agir. Reprenant les travaux d'Alex Khasnabish $^{10}$, Geoffrey Pleyers et Marlies Glasius proposent de décrire les "mouvements de crise " comme des phénomènes de résonance qui produisent des significations politiques de par les rencontres erratiques dont ils sont le moteur : « Il s'agit pour nous d'un lien social qui se situe au plan de l'expérience des activistes et des enjeux et valeurs des mouvements partagés par des activistes, lesquels ne s'engagent pas directement dans une lutte dans un contexte éloigné, mais trouvent dans cette lutte un sens partagé une culture politique et des enjeux qui correspondent à leurs propres luttes $^{11} »$.

\section{Des mobilisations incrémentales}

Depuis les travaux de Charles Tilly ${ }^{12}$ et Doug McAdam ${ }^{13}$, l'une des « lois » robustes de la sociologie de l'action collective, pose que plus existent des liens forts entre les protestataires, leurs amis et leurs connaissances, plus il y a de chances que ces derniers en viennent à s'engager également. Par ailleurs, un certain nombre de chercheurs ${ }^{14}$ estime que lesdits liens forts deviendraient, aujourd'hui, plus rares et plus fragiles, à l'instar des espaces publics physiques (places publiques, bars, cafétérias d'entreprise, etc.) qui en étaient les principaux supports. Quand les TNIC ne sont pas purement et simplement considérées comme un facteur aggravant de cette délitescence du capital social ${ }^{15}$, elles sont parfois, a contrario, appréhendées comme permettant de pallier cette supposée perte d'une sociabilité - notamment politique - composée de «strong ties». 
Plus raisonnablement, les technologies de l'Internet sont également décrites, non pas comme remettant en cause l'exactitude du "binding process » que nous décrivions en ouverture de ce paragraphe, mais comme révélatrices du fait que l'engagement dans une action contestataire peut aussi être indexé à des formes relevant davantage d'un "bridging process", articulant aussi un nombre non négligeable de liens faibles. Pour Rodrigo Nunes, par exemple, le phénomène ne relève pas « d'une simple vague de fond qui permettrait l'augmentation et l'intensification des liens, mais d'un mouvement plus complexe dans lequel les clusters de liens forts et la cohérence organisationnelle qu'ils offrent deviennent essentiels à la structuration de la contagion en ligne et de ce qui se passe sur le terrain ${ }^{16}{ }^{\prime}$.

6 Le rôle que jouent les liens forts semble donc rester essentiel dans la mesure où c'est à partir de ces derniers que se construisent, par cercles concentriques et intégration progressive aux communautés et aux identités les plus affirmées, des collectifs mobilisés. Ces liens forts peuvent préexister à la constitution des communautés en ligne, mais ils peuvent aussi être la conséquence d'un travail spécifique mené sur Internet ${ }^{17}$. Par ailleurs, ils se trouvent enchâssés dans des réseaux de liens plus nombreux, mais plus faibles qui posent, eux aussi, à nouveaux frais, la question de la construction de la confiance au sein de dispositifs médiatisés qui ne permettent qu'une réassurance ténue de la communauté quant à la volonté de participer de ses membres. Outre les posts qui peuvent toutefois être essentiels dans l'établissement explicite d'une certaine assurance quant au déroulement positif de la mobilisation, ce sont surtout, sur les SNS, des indicateurs quantitatifs qui jouent ce rôle de certification des intentions des autres; à l'instar des pages événements de Facebook qui comptabilisent les personnes déclarant vouloir participer ou être intéressées et construisent «l'impression, ou du moins l'illusion nécessaire, qu'il y a un enthousiasme derrière l'événement et que l'on ne finira pas accompagné seulement de quelques autres personnes face à des forces de police écrasantes ${ }^{18}$ ».

7 Nourris du travail de Chantal Mouffe et d'Ernesto Laclau, Paolo Gerbaudo ${ }^{19}$ ou Jeffrey Juris $^{20}$ estiment que les TNIC soutiennent des manières nouvelles de faire collectif, par agrégation: «En l'absence d'identités embarquées et de l'existence de réseaux sociaux denses permettant de soutenir l'action collective, ces éléments ont besoin d'être créés pro-activement et de manière ad hoc dans le cours du processus de mobilisation ${ }^{21}$ ». Aux opérations de réticulation de collectifs (networking ${ }^{22}$ ) censées, par exemple, caractériser les engagements typiques du mouvement altermondialiste, Gerbaudo préfère mettre l'accent sur ce qu'il estime être, aujourd'hui, le plus remarquable, à savoir les processus de "montage" (assembling) de sujets individuels pouvant avoir des backgrounds militants, politiques et idéologiques fort disparates, mais dont l'agrégation est doublement assurée : d'une part, par des rassemblements publics in situ et, d'autre part, par la constitution de communautés en ligne. Par exemple, si le mouvement Occupy s'est appuyé sur les outils largement utilisés par les générations antérieures de militants (mail, listes de diffusion, sites Web, etc.), il a également mobilisé les dispositifs du Web 2.0 (Wiki, Twitter, Facebook, MeetUp, Reddit, Tumblr, Mumble ${ }^{23}$, etc.), ainsi que les médias alternatifs tels que Diaspora, Riseup, The Global Square, Occupii, etc. Cet ensemble de ressources a permis que se développent des pratiques utiles à la mobilisation que Christian Fuchs $^{24}$ décrit comme relevant de dimensions à la fois cognitives, communicationnelles et collaboratives, mais dont il relativise l'importance - sans en dénier pour autant l'effectivité -, en insistant notamment sur le rôle 
prépondérant des conversations en face-à-face (street-level communication), notamment dans la dissémination des informations sur le mouvement. Les SNS semblent néanmoins tout particulièrement prisés car ils donnent accès à de larges publics et facilitent les logiques de diffusion virale dans la mesure où leurs usages, parce qu'aisés, tendent à devenir communs à une partie de la population toujours plus importante. Contrairement aux dispositifs militants qui, même s'ils s'ouvrent en leurs entours, restent globalement fréquentés par des communautés relativement restreintes, les sites de réseaux sociaux portent notamment des audiences nettement moins spécialisées, allant au-delà des cercles militants convenus, constituées pour l'essentiel de liens faibles, mais potentiellement beaucoup plus étendues :

«Dans l'ensemble, alors que les médias sociaux étaient importants pour rassembler une base élargie des jeunes gens issus des classes moyennes, la communication de rue a, elle, permis à ces mouvements de se développer et de devenir véritablement populaires en brisant la fracture numérique et en permettant aux membres de la soi-disant "génération Internet" de s'engager auprès de "ceux qui n'ont pas de compte Facebook" ${ }^{25}$ ".

8 Pour Gerbaudo, les médias numériques attestent ainsi de l'extension de la manière dont les groupes se forment au sein de nos sociétés supposées fragmentées et dispersées, en relation étroite avec un processus complexe de médiations symboliques et techniques qui en assure certaines des conditions de possibilité :

« De tels procédés sont en outre facilités par l'exploitation des hashtags Twitter qui ont le pouvoir de rassembler une communauté d'intérêt autour d'événements et de thèmes mis à jour et identifiés comme les plus pertinents par les membres de cette communauté. Utilisés de cette façon - et il convient de noter que les hashtags peuvent avoir d'autres fonctions que des marqueurs thématiques - les hashtags sont en mesure d'aider à la formation des publics ad hoc sur Twitter; ces publics ad hoc, à leur tour, représentent un sous-ensemble d'un public plus large qui relève de l'écologie globale des médias et de la société elle-même. [...] Ces publics sont dynamiques, leur durée de vie est fonction de la longévité de l'intérêt porté au thème considéré et des contributions et interactions continues entre les membres de ces publics ${ }^{26} »$.

\section{Chorégraphier la lutte}

9 Aussi, «Les formes contemporaines de communication contestataires conduiraient à un élargissement des pratiques de "chorégraphie" : le "scénario" et la "scénarisation" de l'assemblage physique des personnes dans l'espace public "27 ". Pour leur part, Bennett et Segerberg ${ }^{28}$ avancent l'idée que les dynamiques organisationnelles des mouvements sociaux se modifient dès lors que les médias numériques occupent un rôle central dans la mobilisation collective. Le remplacement des formes et des structures hiérarchisées de l'action collective traditionnelle profite, selon eux, à la communication qui devient alors la forme de base de toute organisation collective. Les TNIC permettraient notamment un renouvellement et une flexibilisation des formats d'intégration au mouvement auquel les individus engagés pourraient s'adapter plus aisément en fonction de leurs disponibilités. Ils offriraient par ailleurs la possibilité de promouvoir croyances, convictions et valeurs sans avoir à se soumettre aux procédures hiérarchisées de la prise de parole régulant généralement la diffusion des opinions. Bennett et Segerberg proposent ainsi la notion de "connective action" - qu'ils considèrent comme complémentaire à celle d'action collective ${ }^{29}$ - afin de rendre compte de ce type de modalités d'organisation fondées sur les usages de l'informatique 
connectée et qui autorisent la formation de collectifs erratiques tournés vers l'action, y compris en partant de communautés initialement peu intéressées par la chose politique $^{30}$. À partir d'une recherche reprenant l'appareillage conceptuel de Bennett et Segerberg et s'appuyant sur une enquête empirique portant sur 77 manifestations de masse qui ont eu lieu dans 9 pays européens entre décembre 2009 et juin 2012, Camilo Cristancho et Eva Anduiza ${ }^{31}$ montrent, d'une part, que les mobilisations qui s'appuient sur les SNS tendent à regrouper une large part de personnes qui n'adhèrent à aucune organisation et, d'autre part, que celles-ci font sortir dans la rue des individus qui ne sont pas nécessairement celles et ceux qui battaient jusqu'alors le plus facilement le pavé: "Plus de jeunes, de chômeurs, moins de personnes ayant une implication formelle dans la vie associative, [des individus ayant] des niveaux d'identification plus faibles avec les collectifs organisant les manifestations [et ayant aussi] des niveaux inférieurs de participation politique ». Le rapport au politique et à l'action se créerait davantage via le partage d'expériences et d'événements que par le biais d'appartenances à des organisations militantes ou à des minorités actives.

Henry Jenkins ${ }^{32}$ donne ainsi l'exemple de la communauté Harry Potter Alliance (HPA) dont les membres se servent du contenu fictionnel de l'œuvre de J. K. Rowling pour comprendre les problèmes sociopolitiques du monde qui les entoure et vont parfois jusqu'à s'impliquer dans des actions politiques. Le réseau de fans, par ailleurs entretenu et animé par une lourde équipe de salariés, permet de construire des problèmes publics, d'identifier les acteurs impliqués dans les situations considérées comme problématiques et de mobiliser les jeunes membres de l'Alliance qui acquièrent ainsi des compétences politiques à partir d'un intérêt pour une "culture fan » qui, restant essentielle, sert notamment de sas d'entrée motivationnel à une politisation «de surcroît » pour le moins inattendue. HPA dispose, actuellement, de plus de 100000 membres réunis et distribués en fonction des chapitres du best-seller auxquels ils " adhèrent » et à partir desquels ils sont notamment mis en lien avec un panel varié d'organisations citoyennes qu'ils peuvent soutenir, par exemple financièrement, à l'instar de cette collecte réussissant à lever 123000 dollars qui ont servi à affréter cinq avions transportant des médicaments de première nécessité à destination d'Haïti. La culture populaire « de masse " pourrait ainsi être le support permettant la construction d'une culture populaire "de résistance ». Internet permet de les combiner, facilitant ainsi le passage d'une vision du monde non-élaborée à une culture critique ou oppositionnelle du présent. La possibilité de l'action, et notamment de la conversation/ discussion, concomitante à la production de l'information permet de relativiser le caractère fétiche des culturèmes de masse qui, s'ils tentent d'imposer les signes, les idées, les normes et les désirs correspondant aux conditions concrètes de la marchandisation, peuvent être, au sein même de leur espace de circulation, déconstruits et réappropriés. Ce potentiel de redéfinition et d'assimilation critique d'une culture mainstream, qui par ailleurs peut continuer à être appréciée, pose, d'une part, que les processus contre-hégémoniques ne sont pas nécessairement le fait d'une élite culturelle, tant s'en faut, et, d'autre part, que les formes de résistance peuvent reposer sur des culturèmes, des appétences, des aversions, des rapports au travail et aux loisirs issus à la fois de traditions culturelles anciennes, des industries culturelles, de l'innovation technologique et d'un projet de dépassement de ces héritages.

Cette complexité n'est toutefois pas toujours au cœur des réflexions dédiées. Pour Manuel Castells ${ }^{33}$, par exemple, "la véritable transformation révolutionnaire» des 
politiques du conflit médiées par Internet tiendrait à ce que le changement social émergerait de plus en plus, non d'objectifs programmatiques, mais des "expériences " des individus organisés en réseaux, tant en ligne qu'hors ligne. On peut toutefois être un tant soit peu sceptique devant ce type d'affirmation qui, si l'on prend en considération des mouvements sociaux plus directement politiques, tend à faire l'impasse sur ce que la production et le maintien de la conflictualité sociale suppose en termes de ressources ad hoc à mobiliser et de l'existence d'un capital militant qui, s'il n'est pas distribué entre tous les individus mobilisés, reste nécessairement présent au sein de la frange souvent la plus active et impliquée de ces personnes et qu'ils héritent le plus souvent de trajectoires biographiques les ayant déjà fait traverser d'autres expériences militantes (syndicales, partidaires, etc.) plus « classiques ».

Par ailleurs, quand les "mouvements de crise" sont amenés à quitter l'espace des mouvements sociaux ${ }^{34}$ pour investir le champ politique via, notamment, leur participation à des échéances électorales, apparaissent au grand jour des difficultés qui viennent quelque peu casser le " rythme de la pulsation d'une nouvelle forme de révolution ${ }^{35} »$. Cette autre manière de faire (de la) politique rappelle les conditions concrètes d'instauration de rapports de force indispensables à l'implémentation des changements sociaux, autrement que sous l'égide d'abstractions telles que «la perméabilité des institutions" ou encore "la volonté des mouvements sociaux à s'investir dans des processus de négociation ${ }^{36}$ ». De facto, on risque le malentendu à vouloir trop rapidement confondre la démocratisation de l'espace public numérique et des campements avec les procédures de la démocratie représentative. L'émergence de mobilisations d'individus "par le bas » portées par les TNIC, aussi considérables que celles qui ont eu lieu lors des révolutions arabes et des "mouvements de crise ", ne s'articulent pas naturellement à la construction, "par le haut », d'une représentation politique. Alors que la première coordonne ponctuellement des individus, indépendamment du tissu d'organisations du champ politique, la seconde invite à organiser durablement les citoyens en leur demandant de distribuer leur vote à des collectifs politiques délégataires censés les représenter. On comprend, ainsi, que les résultats des élections en Tunisie, en Égypte, en Espagne ou en Grèce aient pu décevoir tous ceux qui considéraient par trop rapidement les mobilisations réussies d'individus politiquement disparates comme permettant de trouver automatiquement un débouché du côté de la représentation politique. Là, coexistent deux formes d'expression de la citoyenneté beaucoup moins superposables que ne le laissent penser les théoriciens des "nouveaux " " nouveaux mouvements sociaux », de la "démocratie liquide ", des " organisations non organisées ${ }^{37}$ ", tout comme ceux qui considèrent, à l'instar de Gerbaudo, que "c'est la communication qui organise plutôt que l'organisation qui communique ${ }^{38} »$. En témoignent les difficultés qu'ont rencontré les oppositions progressistes du « Printemps arabe » ou les Indignados, dont les velléités électorales les ont mis face à des nécessités qu'ils n'ont su complètement relever ou qui les ont fait se transformer en des forces politiques coupées d'une partie non négligeable de leurs bases originelles.

\section{Émotions et identités 2.0}

Si la circulation accrue de l'information et la facilitation des communications sont deux des caractéristiques évidentes du renouvellement des manières de faire mobilisation, 
les technologies de l'Internet jouent également un rôle dans la production, l'entretien et la propagation des émotions ${ }^{39}$, lesquelles influent notamment sur l'expression des motivations des personnes à prendre part à l'action. Jusqu'à une date récente, le thème des émotions accompagnant les divers moments des politiques du conflit n'a été que peu couver $^{40}$. Les travaux récents de James $\mathrm{M}$. Jasper montrent pourtant toute l'importance de l'énergie émotionnelle, c'est-à-dire de "l'atmosphère d'excitation et d'enthousiasme entretenue autour d'une identité commune ${ }^{41} »$ qui devient alors une ressource essentielle dans le déroulement d'une mobilisation. Ceux de Christophe Traïni soulignent également en quoi l'émotion est un ressort essentiel de certaines mobilisations, à l'instar de la défense de la cause animale, des luttes pour ou contre l'avortement ou encore celles portées par les malades du sida ${ }^{42}$. La présence toujours plus importante des TNIC et la possibilité qu'elles ouvrent quant au partage des événements collectifs et des sensibilités individuelles a toutefois permis de rebattre quelque peu les cartes et de prendre la mesure des ressources émotionnelles pour créer des " chocs moraux ${ }^{43}$ », mais aussi, plus ordinairement, pour " orienter les gens vers des manifestations spécifiques, fournir aux participants des suggestions et des instructions sur la façon d'agir et construire une narration émotionnelle afin de rendre possible leur rencontre dans l'espace public ${ }^{44} »$. Une analyse des tweets durant les émeutes londoniennes de 2011 montre, par exemple, que Twitter a été principalement utilisé pour notifier aux internautes les événements à venir - plutôt que de promouvoir des actions illégales ${ }^{45}$. S'agissant de la page "Democracia Real Ya» des Indignados, Gerbaudo estime qu'elle peut être décrite comme le lieu permettant une accumulation d'énergie émotionnelle " capable de motiver les gens, de les faire "sauter dans la rue" et de les faire surmonter leur isolement et leur passivitée ${ }^{46}$ ». Au fait que les collectifs des "mouvements de crise " sont présentés comme étant spontanés, sans leader et ouverts à toutes les contributions, s'ajoutent les invitations à commenter, aimer et partager les informations qui sont incessantes. Les mises à jour sont particulièrement nombreuses, tout comme les réponses rapides apportées aux commentaires, qui donnent l'impression aux contributeurs de participer pleinement à une communauté d'échanges, mobilisée et partageant des valeurs identiques.

Les messages produits sur les SNS « reflètent la construction d'un effort émotionnel (emotional striving) pour éloigner les activistes d'un sentiment d'isolement, de dispersion et de passivité que ceux-ci considèrent comme la marque de l'expérience sociale des régimes autoritaires [...], mais aussi celle du néolibéralisme qui s'attaque à toutes les formes d'espace public. Devant cette situation de crise de l'espace public, les SNS sont devenus des canaux émotionnels permettant de reconstruire un sentiment de cohésion entre des individus spatialement dispersés et de faciliter leur rapprochement physique dans l'espace public. [...] [Les activistes numériques] ont ainsi utilisé les SNS pour construire une tension émotionnelle, en tirant un pont entre les interactions très dispersées et individualisées que les gens maintiennent via [les SNS] et l'immersion corporelle des rassemblements collectifs. Ces formes de communication ont contribué à créer un sentiment contagieux d'anticipation et d'élan (impetus) avant les manifestations et une attraction émotionnelle autour de la participation aux sit-in de masse. [...] Les SNS peuvent [ainsi] être utilisés pour construire un sentiment de solidarité au sein d'une communauté hétérogène partageant un sentiment commun d'indignation, de colère, de frustration [...]. Les médias sociaux deviennent le pôle d'agrégation ou, mieux encore, de concentration, de ces sentiments individuels, en les transformant en passions politiques dans un récit d'unité populaire. [...] Le rôle le plus important des SNS réside dans la construction d'un sentiment émotionnel de solidarité parmi les participants dispersés, plutôt que dans la coordination des opérations de combat sur le terrain. 
[...] On peut d'ailleurs supposer que les mouvements contemporains pourraient se débrouiller assez bien sans les moyens tactiques offerts par les SNS. Mais ce dont ils ne peuvent pas se passer (ou ce qu'ils font mieux avec), tient à la capacité des SNS à devenir les instruments d'une narration émotionnelle capable de motiver l'individu à se mobiliser dans la rue ${ }^{47}$ ".

14 Les TNIC semblent donc favoriser la constitution de communautés pré-politiques à travers le partage d'émotions, d'intérêts et de valeurs. La possibilité de suivre les événements en temps réel, celle de pouvoir garder traces des événements majeurs, de publiciser les "ressentis de la lutte", tout comme de pouvoir échanger avec une communauté élargie de personnes intéressées ou impliquées dans la mobilisation, contribue en effet à créer ou maintenir des sentiments spécifiques, d'appartenance ou d'activation des engagements (affective synchronisation ${ }^{48}$ ). La chose est par exemple observable dans le cas des groupes Facebook créés après les attentats du 7 janvier de 2015 visant les bureaux parisiens du journal Charlie Hebdo et ayant causé la mort de douze personnes, dont huit collaborateurs du journal satirique. Deux groupes de soutien "Je suis Charlie » et "Nous sommes Charlie » ont alors été montés par des individus isolés, récoltant rapidement, pour l'un, près de 91000 membres et pour l'autre plus de 200 000. Le succès de ses communautés qui vont, par la suite, porter des mobilisations hors ligne tient, selon Romain Badouard, au fait qu'elles permettent aux membres du public de s'approprier l'événement et d'exprimer une émotion à la première personne. Elles offrent aux "prenant-parts " l'agrément d'une nouvelle perception du monde à partir d'un Je qui se transforme en Nous, en s'appuyant sur des émotions individuelles : «Le recours à la première personne correspond également à la manière dont se structurent les mouvements d'opinion et les actions collectives sur Internet et les réseaux sociaux : ceux- ci présentent toujours une dimension individuelle, puisque les internautes sont seuls devant leur écran, et la mise en commun d'une émotion ou d'une indignation relève toujours d'un processus d'agrégation de prises de parole isolées ${ }^{49}{ }$.

La mise en visibilité et en publicité des causes et des mobilisations, accompagnée d'une expression publique de leurs dimensions sensibles (souffrances, résignations, colères, etc.) tend notamment, pour certains chercheurs ${ }^{50}$, à faciliter le passage de l'indignation personnelle à l'action individuelle et de cette dernière à l'action collective, en maintenant un « sens du collectif » qui se nourrirait « de la tension émotionnelle de la connexion à distance [jusqu']à l'effervescence de la proximité physique ${ }^{51} »$. Les travaux empiriques portant sur le caractère "performatif » des médias numériques restent toutefois relativement évasifs quant aux mécanismes précis reliant publicisation/ réception des émotions et engagement dans l'action. Ils se fondent, le plus souvent, sur des explications mécanistes, linéaires et médiacentristes, à l'instar des recherches de Castells qui estime que l'existence d'une " culture Internet » aurait été la condition de possibilité à l'émergence des révoltes ou de celles de Juris qui stipule que les logiques agrégatives des mouvements Occupy sont directement empruntées aux scripts des sites de réseaux sociaux. Les études de cas les plus précises portent, en ce domaine, sur les révolutions arabes et notamment les "Printemps" tunisien et égyptien. Elles montrent, par exemple, que la mobilisation autour des pages Facebook «Kullena Khaled Saïd » a permis que se construisent des formes de "condensation émotionnelle » autour de "signifiants vides » et neutres (empty signifier), à partir desquels une unité performative peut émerger. Wael Ghonim témoigne ainsi : «Dès les premiers jours, 36 000 personnes rejoignent la page. [...] C'est l'image de la mort atroce de Khaled, ainsi 
que son appartenance à la classe moyenne, qui catalysent cette énorme réaction. La photo est impossible à oublier, et grâce aux réseaux sociaux, elle prolifère. À la fin du premier jour, il y a plus de 1800 commentaires sur la page ${ }^{52}$ ». Ulrike Riboni montre, pour sa part, que les "stratégies visuelles ", tout au long du processus révolutionnaire tunisien, notamment celles prenant en charge le traitement médiatique des blessés et des martyrs opèrent largement sur le registre émotionnel. Analysant des vidéos donnant à voir la répression subie par les habitants de Redeyef, elle identifie deux registres iconiques: les images-preuve, "destinées à servir de preuve de l'usage de lacrymogènes, de balles réelles ou de violences sur les personnes » et les images-émotion "destinées à galvaniser [et à rendre] compte d'un sentiment d'oppression et de danger $\aleph^{53}$. Et Cécile Boëx d'estimer que dans le cas de la Syrie,

«la mise en images des manifestations contribue également à façonner des dispositifs de sensibilisation spécifiques, dont l'objectif est de susciter des réactions affectives promptes à rallier un soutien. Ces dispositifs permettent une réappropriation de l'espace public, tout comme ils constituent des vecteurs puissants d'un imaginaire protestataire articulé autour du paradigme du courage. Slogans, chants et danses sont autant de techniques pour faire corps et démontrer la cohésion ainsi que la détermination du collectif, tout en communiquant une charge émotionnelle. Ces vécus émotionnels et leurs modes de communication sont d'autant plus forts et efficaces qu'ils exposent également, en creux, le sacrifice consenti ${ }^{54} »$.

\section{De la construction des identités}

La question de l'émotion est par ailleurs intimement liée à celle de la construction des identités. Depuis la fin des années 1960, la problématique identitaire a été largement mobilisée au sein de la sociologie de l'action collective, notamment afin de rendre compte des liens qui se tissent entre les militants mobilisés, mais aussi des motivations à s'engager au sein d'un mouvement. L'émergence de ce que l'on a appelé les "nouveaux mouvements sociaux " (NMS), portant des combats autres que ceux découlant de la contradiction capital/travail (féminisme, immigration, écologie, homosexualité, etc.) et s'appuyant sur des identités autres que celles de classes ${ }^{55}$, a entrainé la conduite de recherches relatives à une série de mobilisations politisant des enjeux qui étaient, auparavant, largement confinés à la sphère privée, et valorisant l'expérience des sujets ${ }^{56}, \mathrm{y}$ compris parfois dans ce que celle-ci peut avoir de plus intime (identity-oriented). L'étude des NMS a donc permis de saisir les processus de construction identitaire comme une composante essentielle des luttes sociales, lesquels ont aussi été étudiés au sein des travaux les plus récents. Certains chercheurs voient en effet, dans les "mouvements de crise", une actualisation des dynamiques qui étaient celles des NMS, dans la mesure où les identités citoyennes en jeu au sein de ces luttes semblent fonctionner comme des ressorts affectifs et fournir aux militants des ressources expressives "encapacitantes ${ }^{57}$ ». Ces travaux mettent notamment en lumière que la formation des identités militantes se nourrit assez largement des pratiques partagées en ligne par les internautes mobilisés. On sait maintenant, depuis des décennies, que l'intérêt à l'engagement est motivé par des paramètres psychoaffectifs ${ }^{58}$, une réassurance identitaire, la recherche de solidarités ${ }^{59}$ et le gain d'un ensemble de rétributions symboliques ${ }^{60}$. Ces satisfactions spécifiques solidaires de la figure du militant investi classiquement dans une organisation semblent pouvoir être, au moins partiellement, prises en charge par certaines activités de discussion, de 
commentaire, de partage de contenus, d'expériences et d'émotions. Réitérées et se transformant en habitudes, celles-ci permettent l'émergence de sociabilités singulières et de sentiments d'appartenance; à l'instar de ce qui peut se passer sur Avaaz ou Facebook, où les formes d'interactivité et de réciprocité se trouvent au fondement du développement d'identités collectives.

17 Angela Suarez Collado ${ }^{61}$ montre ainsi comment Internet a pu faciliter l'expression de l'identité amazighe (berbère), permettre l'expression des intellectuels organiques de cette communauté et autoriser la conservation du patrimoine immatériel de celle-ci (proverbes, musique, etc.). Certaines fêtes sont documentées, filmées et mises en ligne, les personnages historiques imazighen sont valorisés, les traditions culturelles sont présentées comme des repères transnationaux susceptibles de lier la communauté pardelà les frontières. En ce cas, Internet permet également de renforcer les singularités communautaires régionales au principe desquelles sont nées des revendications autonomistes, par exemple dans le Rif ou le Sousse. Au surplus, les espaces de discussion ouverts sur le Web (blogs, forums, groupes Facebook, etc.) contribuent à ce que se renforce une conscience commune à partir de laquelle les actions protestataires de rue - à l'instar du « Mouvement du 20 février 2011 »- deviennent possibles et leur coordination plus commode à conduire. Autrement dit, les technologies de l'Internet permettent d'appareiller les différentes dimensions identitaires telles que définies par Verta Taylor et Nancy Witthier ${ }^{62}$ : l'établissement de frontières distinguant un "Nous » et un «Eux» et amenuisant les différences au sein même de la minorité mobilisée ; l'activation d'une mémoire populaire en résistance ouvrant à un exercice de réflexivité et de réévaluation positive de traditions (culturelles, de lutte, etc.) ; la redéfinition des cadres hexogènes de définition des singularités du collectif. Dans un tout autre domaine, une ethnographie du média alternatif Attac-info ${ }^{63}$ montre, par exemple, qu'il s'agit d'un collectif dont le potentiel «se prépare et s'alimente dans la situation de latence ${ }^{64}$ ». En effet, le rassemblement physique des militants épouse les phases successives de convergence et de mise en veille caractérisant le cycle de mobilisation $\mathrm{du}$ mouvement altermondialiste lors des forums sociaux. Aussi, le groupement n'acquiert de la permanence et ne maîtrise son identité collective que dans la mesure où un effort est effectué pour entretenir certaines relations affectives, expertes et/ou militantes dans l'intervalle des mobilisations. Et Internet se présente comme un élément majeur de structuration de la vie du collectif, permettant de maintenir « une série de liens [qui deviennent] explicites seulement à l'occasion des mobilisations collectives sur des enjeux à propos desquels le réseau latent remonte à la surface, pour ensuite s'immerger à nouveau dans le tissu du quotidien ${ }^{65}$ ".

18 L'usage des TNIC renforce également une expressivité qui fait droit à la particularité et à l'autonomie des individus en leur permettant de mettre en visibilité des causes et des identités personnelles particulières - notamment celles qui peuvent se trouver parmi les plus stigmatisées ou dévaluées par les institutions politiques et médiatiques dominantes (Denouël et al., 2014). Par conséquent, les repères communautaires servent également de terreau à des formes d'affirmation de soi liées à des demandes explicites de reconnaissance ${ }^{66}$. Se défiant des "totalités militantes", les internautes mettent en avant leurs expériences et leurs qualités personnelles via la production d'expressions citoyennes qui peuvent être appréhendées comme des formes d'accomplissement pratique dont l'un des ressorts est celui du "self at source ${ }^{67} »$. Singularités identitaires et intérêt général se côtoient. Autrement dit, les internautes engagés se mobilisent à 
partir d'un double motif qui relève à la fois d'un sens de la réalité tenant à l'intérêt public et à un processus identitaire. Ces modes de production de soi s'appuient sur la mise en visibilité de traits distinctifs davantage attachés au sujet (goûts, descriptions d'activités triviales, échanges avec des proches, etc.) tout en revendiquant la participation citoyenne à des communautés solidaires de pensée et de débat. Dans un style qui n'emprunte plus guère aux sciences sociales, Castells écrit par exemple : «Les racines d'une nouvelle vie se répandent partout, sans plan central, se déplaçant et se réticulant, conservant la fluidité de l'énergie, en attendant le printemps. Parce que ces nœuds sont toujours connectés. Il y a les nœuds des réseaux de l'Internet, locaux et mondiaux, et il y a les réseaux personnels, vibrant sous l'impulsion d'une nouvelle sorte de révolution dont l'acte le plus révolutionnaire est l'invention de soi $^{68}{ }^{\prime}$. Si le constat ainsi formulé apparaît quelque peu désincarné, il est toutefois confirmé par de nombreux travaux ${ }^{69}$ que l'investissement dans la production d'expressions citoyennes qui, à un premier niveau, relève d'un engagement civique, se double également de formes de production de soi dont l'importance peut fortement varier d'un sujet à l'autre. La participation à l'espace numérique par le biais de la production symbolique de contenus informationnels de portée publique vient en fait s'arrimer à des dynamiques de construction d'identités narratives ${ }^{70}$ qui semblent constituer une part non négligeable des ressorts motivationnels des blogueurs et utilisateurs de SNS.

«Les révoltes des années 2010 [mêlent] profondément les revendications économiques, sociales, politiques et culturelles et les combinent avec une forte dimension éthique. Elles sont à la fois profondément personnelles et globales, ancrées dans des enjeux locaux et nationaux, tout en s'inscrivant dans une vague mondiale de mobilisation. [...] L'engagement d'aujourd'hui n'y est pas que social et collectif. Il est aussi profondément personnel. L'implication dans un mouvement social travaille profondément l'individu jusque dans sa subjectivité et sa subjectivation, entendue comme la manière de se penser et de se construire soimême comme principe de sens ${ }^{71}$ ".

19 Le recouvrement d'intérêts à la fois généraux et personnels enjoint à trouver des équilibres singuliers entre ce que les internautes proposent et ce qu'ils sont, mais également entre leurs activités en ligne et leur vie hors ligne. Si d'aucuns passent par le pseudonymat et travaillent à la séparation étanche des pratiques, d'autres, au contraire, cherchent l'intégration publique de leurs activités et de leurs identités. La réussite et la pérennité de ces entreprises semblent ainsi tenir à la gestion de stabilités multiples entre l'individuel et le collectif, le singulier et le général, le online et le offline, etc., qui dépendent notamment des interactions avec les publics. Il s'agit donc de mettre en œuvre des compétences particulières, socialement différenciées, concernant l'aptitude à maintenir une compatibilité entre des formes symboliques à forte charge civique et des facettes singulières de soi dans un espace de parole composite liant des publics susceptibles de ratifier ces deux aspects. Dans ce domaine, l'économie de moyens et la structure potentiellement virale de la circulation de l'information sur Internet, constituent alors un moteur décisif pour ces formes nouvelles de mobilisations informationnelles.

20 S'agissant des internautes tunisiens, Romain Lecomte note qu'expressions de soi et paroles politiques ont été fortement enchevêtrées : «Un grand nombre de blogueurs [...] alternent [...] des billets consacrés à leur vie privée et leur intériorité, d'autres à un thème spécifique qu'ils affectionnent particulièrement [...] et d'autres encore à des questions d'intérêt général, des questions sociétales et politiques ${ }^{72}$ ». 
21 Et d'ajouter : «Un exemple illustrant la variété des formes d'énonciation en public portées par un(e) même internaute est celui de la célèbre blogueuse Lina Ben Mhenni (professeur d'anglais à l'Université de Tunis) : celle-ci, sur son blog et sur ses comptes Facebook et Twitter, alterne et même souvent entremêle depuis plusieurs années, dévoilement de son intériorité et de sa vie privée (sentiment de déprime, prise de médicaments, envies de "se faire belle", relation entretenue avec son père, etc.) et expression politique. Même lors des mobilisations protestataire de l'hiver 2010-2011, que Lina a couvertes intensivement (se rendant également sur le terrain), la blogueuse a continué à affirmer sa subjectivité et se mettre en récit ${ }^{73}$ ».

Les travaux effectués sur les "mouvements de crise » semblent confirmer l'hypothèse selon laquelle les identités singulières se visibilisant en ligne sont, elles aussi, marquées par des demandes de reconnaissance. La circulation de l'information en ligne facilite l'échange d'expériences, de sentiments, de valeurs, d'opinions et, partant, l'invention de nouvelles formes d'identités politiques ${ }^{74}$. Celles-ci se modulent autour d'attendus citoyens qui tiennent moins à un accord idéologique préalable ou à des convictions communes unanimement ratifiées, qu'au partage d'une culture civique ${ }^{75}$ élargie qui emprunte diversement à l'idéal participatif, à l'impératif d'information, ainsi qu'à la nécessité du débat public.

«Par exemple, depuis 2006, de nombreux blogueurs réalisaient chaque 25 décembre une "note blanche" pour défendre la cause de la liberté d'expression et en particulier pour dénoncer la censure d'Internet: ils publiaient sur leur espace personnel un billet avec pour titre "Note blanche", mais dépourvu de contenu. [...] ce type d'actions, parallèlement à leur dimension protestataire (parfois très ténue), avait également une dimension rituelle : elles contribuaient à l'expression et la consolidation d'une "communauté" de blogueurs tunisiens ${ }^{76}$ ".

Les revendications des Indignés dénoncent par exemple la violation des principes de justice et d'égalité, cadrage large d'expressions politiques qui permet une ouverture maximale à l'intégration de points de vue hétérogènes qui trouvent par ailleurs, sur Internet, le moyen de s'exprimer. C'est ainsi, par la stimulation d'un imaginaire commun de résistance au pouvoir, le ralliement à des valeurs civiques générales, le tout mêlé à des demandes de reconnaissance qui trouvent à s'actualiser en ligne, que des sentiments d'appartenance se créent ${ }^{77}$. De facto, l'usage de l'informatique connectée permet aux divers contributeurs de bénéficier auprès de publics variés d'un ensemble de rétributions symboliques qu'ils n'auraient pu obtenir dans l'exercice "classique » d'un militantisme où la valorisation du travail tient davantage à la mise en œuvre de compétences politiques et organisationnelles qu'ils ne maîtrisent pas nécessairement. Peter Dahlgren ${ }^{78}$ estime qu'Internet permet de sortir les expressions citoyennes de la normativité de la sphère publique et des secteurs militants et partisans pour les indexer davantage à l'idéal de la subjectivité, de la communication et de la sociabilité : «Les identités militantes ne sont pas données mais sont constituées dans le processus d'action collective en ligne ${ }^{79} »$. Elles sont le précipité des engagements, sourdent des investissements singuliers qui se concatènent, mais ne leur préexistent pas. Vivre le collectif et partager un entre-soi n'est plus une condition préalable de l'action, mais en est plutôt le résultat. Aussi apparaît-il difficile de créer un "Nous" pleinement mobilisateur quand il se compose $\mathrm{d}^{\prime}$ ' individus aux engagements éphémères, résiliables à tout moment, limités dans le temps comme dans la tâche à accomplir ${ }^{80}$ ". Certains chercheurs soulignent alors combien ces identités collectives pour parties nourries d'opérations online génèrent des configurations identitaires instables (transient 
identity ${ }^{81}$ ) à partir desquelles il reste difficile d'élever et de pérenniser des cadres d'action $^{82}$. Natalie Fenton et Veronica Barassi notent à cet effet que "les formes autocentrées de communication que les [sites de] réseaux sociaux génèrent peuvent contraindre plutôt que renforcer la créativité collective des mouvements sociaux ${ }^{83}$ ». Elles suggèrent notamment que les formes particulières d'interaction qui donnent voix aux opinions individuelles, aux niches affinitaires, aux micro-dépendances amicales et aux initiatives de sous-collectifs peuvent, in fine, amoindrir la créativité globale d'un mouvement qui, sur Internet, peut devenir bavard, illisible, incohérent et s'en trouver alors fragilisé, notamment quant à ses potentiels de ralliement et ses capacités de mobilisation.

Nous assistons, aujourd'hui, au niveau mondial, à une dégradation importante des conditions sociales d'existence. Le salariat exploité ne cesse d'augmenter, les politiques d'austérité se multiplient, la crise de 2008 a accru le nombre de travailleurs précaires, pauvres, vulnérables et sans emploi (notamment chez les femmes et les jeunes); la croissance des salaires est bloquée pour les moins dotés économiquement, les déplacements forcés de population s'intensifient, etc. La part des revenus alloués au travail a diminué, tandis que les richesses sont toujours plus concentrées entre les mains d'une oligarchie. À cet abaissement général des conditions de vie des " $99 \%$ ", fait pièce une recrudescence des protestations sociales et politiques. À l'échelle internationale, les syndicats et les mobilisations, sur fond de contradiction capital/ travail, se sont largement développés. Le mouvement altermondialiste a permis que se tissent des liens militants pérennes permettant une coordination plus aisée de certaines causes et luttes. Les "mouvements de crise" ont mobilisé en nombre. L'instabilité politique, les crises de régime et les dysfonctionnements institutionnels des pays émergents, autoritaires, mais aussi des démocraties occidentales vont grandissant.

La liste des points de tension avec l'ordre social dominant est, ici, loin d'être complète, mais on peut globalement avancer que les volontés pratiques de dépassement du capitalisme restent très actives. Elles s'ajustent aux spécificités plus nombreuses des formes d'impérialisme (régionaux, nationaux, continentaux, etc.), et notamment à la nouvelle division internationale du travail. Leur vitalité («Printemps arabe», mouvements d'occupation, grèves ouvrières, mobilisations féministes, LGBTI, écologistes, de migrants, etc.) crée d'ailleurs un raffermissement des réponses du système, lesquelles vont des révolutions passives d'aménagement du Welfare - là où il existe encore -, à la libéralisation accrue des communs, en passant par la mise en œuvre de contre-révolutions violentes, ou encore celle de formes plus classiques de répression et d'offensives réactionnaires (état d'exception, mise à mal des droits sociaux fondamentaux, montée du néoconservatisme, des extrêmes droites, du fondamentalisme, du racisme, etc.). La mondialisation capitaliste fait évoluer les modes de domination de classe, les durcit, mais déclenche aussi un niveau de conflictualité sociale particulièrement important qui semble la plonger dans une instabilité structurelle. D'aucuns estiment que s'ouvre actuellement une séquence de crise permanente (institutionnelle, idéologique, de légitimité), où le niveau d'affrontement social (grèves, manifestations, occupations, insurrections, émeutes, etc.) pourrait devenir particulièrement élevé - i.e. ouvrir des crises révolutionnaires: grèves générales insurrectionnelles, guerres populaires prolongées - et épouser des formes d'engagement et d'action collective de plus en plus variées, tant sur le plan des causes 
qu'au niveau des manières de faire collectif, ou quant à celui des répertoires de mobilisation.

Malgré cette diversité, la plupart des mouvements protestataires ont en commun leur rapport négatif au système capitaliste et à la crise économique mondiale. Ils partagent le fait de dénoncer la démocratie représentative (désobéissance civile, valorisation du consensus, de la démocratie directe, etc.), mais aussi d'utiliser les technologies numériques d'information et de communication à des fins de diffusion, de débat, de mobilisation et de coordination ${ }^{84}$. Davantage qu'une simple infrastructure, fut elle « de résistance ${ }^{85}$ ", Internet tend à redéfinir à la marge, mais aussi de plus en plus centralement, les frontières de l'action collective et la nature des modes de résistance et de contestation. Dès la fin des années 1990 et l'émergence du mouvement altermondialiste, le développement des usages d'Internet a permis que s'actualisent de nouveaux potentiels d'entraide, d'échange d'information, d'expertise et que soit facilitée l'articulation de fronts de contestation au niveau local, national et/ou international. Aussi, Bruce Bimber et ses collègues ${ }^{86}$ avancent qu'Internet ouvre de nouvelles formes de mobilisation dont l'étude permettrait d'éclairer plusieurs aspects fondamentaux de l'action collective qui, jusqu'alors, avaient été peu ou mal théorisés. À l'évidence, décrire, expliquer, comprendre et juger des modalités concrètes de déploiement de la conflictualité sociale oblige aujourd'hui à prendre en considération la dialectique sociotechnique $e^{87}$ qui nourrit les politiques du conflit à l'ère du numérique, laquelle peut être aussi bien habilitante que limitative.

\section{BIBLIOGRAPHIE}

Alhassen (Maytha), Shihab-Eldin (Ahmed) eds., Demanding Dignity: Young Voices from the Arab Spring, Ashland, White Cloud, 2012.

Badouard (Romain), «"Je ne suis pas Charlie” : pluralité des prises de paroles sur le web et les réseaux sociaux », in Lefebure (Pierre), Secail (Claire), Le défi Charlie, Les médias à l'épreuve des attentants, Paris, Le Mieux-Éditeur, 2015, pp. 187-221.

Badouard (Romain), « Les mobilisations de clavier. Le lien hypertexte comme ressource des actions collectives en ligne ", Réseaux, vol. 5, n 181, 2013, pp. 87-117.

Bauman (Zygmunt), The Individualized Society, Cambridge, Polity Press, 2001.

Bennett (Lance), Segerberg (Alexandra), The Logic of Connective Action. Digital Media and the Personalization of Contentious Politics, New York, Cambridge University Press, 2014.

Bennett (Lance), Segerberg (Alexandra), «The logic of connective action: Digital media and the personalization of contentious politics ", Information, Communication \& Society, vol. 15, $\mathrm{n}^{\circ} 5,2012$, pp. 739-768.

Benski (Tova), Langman (Lauren), «The effects of affects: the place of emotions in the mobilizations of $2011 »$, Current Sociology, vol. 61, n 4, 2013, pp. 525-540. 
Bimber (Bruce), Flanagin (Andrew J.), Stohl (Cynthia), « Reconceptualizing Collective Action in the Contemporary Media Environment », Communication Theory, vol. 15, n 4, 2005, pp 365-388. Boëx (Cécile), « La vidéo comme outil de l'action collective et de la lutte armée », in Burgat (Françoise), Paoli (Bruno), Pas de printemps pour la Syrie. Les clés pour comprendre les acteurs et les défis de la crise [2011-2013], Paris, La Découverte, 2013, pp.173-184.

Bruns (Axel), Highfield (Tim), « The Arab Spring on Twitter: Language Communities in \#egypt an \#lybia ", in Bebawi (Saba), Bossio (Diana) eds., Social Media and the Politics of reportage. The «Arab Spring ", New York, Palgrave Macmillan, 2014, pp. 33-55.

Castells (Manuel), Networks of Outrage and Hope. Social movements in the Internet Age, Cambridge/ Malden, Polity Press, 2012.

Chadwick (Peter), Continuum mechanics: concise theory and problems, Londres, Courier Corporation, 2012.

Chambat (Pierre), Jouët (Josiane), « Machines à communiquer : acquis et interrogation », in dixième Congrès National des SIC, Grenoble-Echirolles, SFSIC, 1996, pp. 207-214.

Cristancho (Camilo), Anduiza (Eva), « Connective Action in European Mass Protest », communication aux ECPR Joint Sessions of Workshops Mainz, 11-16 March 2013http:// www.lapietradialogues.org/area/pubblicazioni/doc000075.pdf.

Dahlgren (Peter), The political web: Media, participation and alternative democracy, Londres, Palgrave Macmillan, 2013.

Dahlgren (Peter), « The Internet, public spheres, and political communication: dispersion and deliberation », Political Communication, $\mathrm{n}^{\circ} 22,2005$, pp. 147-162.

Dahlgren (Peter), « Reconfigurer la culture civique dans un milieu médiatique en évolution », Questions de communication, $\mathrm{n}^{\circ}$ 3, 2003, pp. 151-168.

Denouël (Julie), Granjon (Fabien), Aubert (Aurélie), Médias numériques et participation. Entre engagement citoyen et production de soi, Paris, Mare \& Martin, 2014.

Fenton (Natalie), Barassi (Veronica), « Alternative media and social networking sites: The politics of individuation and political participation », The Communication Review, vol. 14, $\mathrm{n}^{\circ} 3,2011$, pp. 179-196.

Fuchs (Christian), OccupyMedia! The Occupy Movement and Social Media in Crisis Capitalism, Winchester, Zero Books, 2013.

Gaxie (Daniel), «Économie des partis et rétributions du militantisme », Revue française de science politique, vol. 27, $\mathrm{n}^{\circ} 1,1977$, pp. 123-154.

Gerbaudo (Paolo), Tweets and the Streets: Social Media and Contemporary Activism, London, Pluto Press, 2012.

Ghonim (Wael), Révolution 2.0. Le pouvoir des gens plus fort que les gens au pouvoir, Paris, Steinkis, 2012.

Goodwin (Jeff), Jasper (James M.), Polletta (Francesca) eds., Passionate Politics. Emotions and Social Movement, Chicago, University of Chicago Press, 2001.

Granjon (Fabien), «Attac-info : entre communauté d'action et espace de représentation. Ethnographie d'un média “alter" lors du Forum social mondial 2003 », Matériaux pour l'histoire de notre temps, $\mathrm{n}^{\circ} 79,2005$, pp. 70-76. 
Granjon (Fabien), L'Internet militant. Mouvement social et usages des réseaux télématiques, Rennes, Apogée, 2001.

Granjon (Fabien), Lelong (Benoît), « Capital social, stratifications et technologies de l'information et de la communication. Une revue des travaux français et anglo-saxons ", Réseaux, vol. 24, $\mathrm{n}^{\circ} 139$, 2006, pp. 147-182.

Halleck (DeeDee), Hand-held Visions. The Impossible Possibilities of Community Media, New York, Fordham University Press, 2002.

Honneth (Axel), La lutte pour la reconnaissance, Paris, Cerf, 2000.

Ion (Jacques) dir., L'engagement au pluriel, Publications de l'Université de Saint-Étienne, SaintÉtienne, 2001.

Jasper (James M.), « Emotions and Social Movements: Twenty Years of Theory and Research », Annual Review of Sociology, $\mathrm{n}^{\circ}$ 37, 2011, pp. 285-303.

Jasper (James M.), The Art of Moral Protest. Culture, Biography, and Creativity in Social Movements, Chicago, University of Chicago Press, 1997.

Jenkins (Henry), «Cultural Acupuncture: Fan Activism and the Harry Potter Alliance », Transformative Works and Cultures, $\mathrm{n}^{\circ}$ 10, 2012. http://journal.transformativeworks.org/ index.php/twc/article/view/305/259.

Jenkins (Henry), « The cultural logic of media convergence », International Journal of Cultural Studies, vol. 7, $\mathrm{n}^{\circ} 1,2006$, pp. 33-43.

Juris (Jeffrey), «Reflections on \#Occupy Everywhere: Social Media, Public Space, and Emerging Logics of Aggregation », American Ethnologist, vol. 39, n² 2, 2012, pp. 259-279.

Juris (Jeffrey), Networking futures: The movements against corporate globalization, Durham, Duke University Press, 2008.

Khasnabish (Alex), « Insurgent Imaginations », Ephemera, vol. 7, n 4, 2007, pp. 505-525.

Khosrokhavar (Farhad), The New Arab Revolutions that Shook the World, Boulder, Paradigm Publishers, 2012.

Klandermans (Bert), « Transient Identities? Membership Patterns in the Dutch Peace Movement ", in Laraña (Enrique), Johnston (Hank), Gusfield (Joseph R.), eds., New Social Movement. From Ideology to Identity, Philadelphie, Temple University Press, 1994, pp. 168-184.

Klandermans (Bert), « Mobilization and Participation: Social-Psychological Expansions of Resource Mobilization Theory », American Sociological Review, vol. 49, n 5, 1984, pp. 583-600.

Latté (Stéphane), «Des “mouvements émotionnels" à la mobilisation des émotions », Terrains/ Théories, $\mathrm{n}^{\circ}$ 2, 2015. http://teth.revues.org/244.

Lecomte (Romain), « Les usages "citoyens" d'Internet dans le contexte autoritaire tunisien : analyse de l'émergence d'un nouvel espace public de la critique », in Najar (Sihem) dir., Le cyberactivisme au Maghreb et dans le monde arabe, Tunis/Paris, IRMC/Karthala, 2013.

Mason (Paul), Why It's Kicking Off Everywhere: The New Global Revolutions, Londres, Verso, 2012.

Mathieu (Lilian), Comment lutter ? Sociologie et mouvements sociaux, Paris, Textuel, 2004.

McAdam (Doug), Freedom Summer, Oxford, Oxford University Press, 1988.

McAdam (Doug), Political Process and the Development of Black Insurgency (1930-1970), Chicago, The University of Chicago Press, 1982. 
Melucci (Alberto), Challenging Codes: Collective Action in the Information Age, Cambridge, Cambridge University Press, 1996.

Melucci (Alberto), « The symbolic challenge of contemporary movements », Social research, vol. $52, \mathrm{n}^{\circ} 4,1985$, pp. $789-816$.

Melucci (Alberto), « Mouvements sociaux, mouvements post-politiques », Revue internationale d'action communautaire, $\mathrm{n}^{\circ} 10,1983$, pp. 13-30.

Melucci (Alberto), « The New Social Movements: A Theoretical Approach », Social Science Information, $\mathrm{n}^{\circ} 19,1980, \mathrm{pp} .199-226$.

Milan (Stefania), Social movements and their technologies. Wiring social change, New York, Palgrave Macmillan, 2013.

Mitrović (Marjira), Paltoglou (Georgios), Tadić (Bosiljka), « Quantitative analysis of bloggers' collective behavior powered by emotions ", Journal of Statistical Mechanics: Theory and Experiment, $\mathrm{n}^{\circ}$ 2, 2011. http://www.scl.rs/papers/Mitrovic_JSTAT_2011.pdf.

Negt (Oskar), L'espace public oppositionnel, Paris, Payot, 2007.

Nunes (Rodriguo), Organisation of the organisationless: collective action after networks, Lüneburg, Mute Books/PML Books, 2014.

Oberschall (Anthony), Social Conflict and Social Movements, Englewood Cliffs, Prentice Hall, 1973.

Offe (Claus), « New social movements: Challenging the boundaries of institutional politics », Social Research, vol. 52, n 4, 1985, pp. 817-868.

Papa (Venetia), Dynamics and Synergies around/and between civic and collective identity and the role of Facebook within the context of Indignados movement, thèse de doctorat, Cyprus University of Technology/Université Paris 8, 2015.

Papa (Venetia), Milioni (Dimitra), « I don't wear blinkers alright? An analysis of civic identity in the Indignados movement and the role of Facebook », Javnost-The Public, vol. 23, n 3, 2016, pp. 290-306.

PLEYERS (Geoffrey), Capitaine (Brieg) dir., Mouvements sociaux. Quand le sujet devient acteur, Paris, Éditions de la Maison des sciences de l'homme, 2016.

PLEYERS (Geoffrey), Glasius (Marlies), « La résonnance des “mouvements des places” : connexions, émotions, valeurs ", Socio, $n^{\circ} 2,2013$, pp. 59-79.

Poster (Mark), Information Please: Culture and Politics in the Age of Digital Machines, Durham, Duke University Press, 2006.

Prentoulis (Marina), Thomassen, (Lasse), « Political theory in the square: Protest, representation and subjectification », Contemporary Political Theory, vol. 12, n 3, 2013, pp. 166-184.

Riboni (Ulrike L.), «Juste un peu de vidéo ». La vidéo partagée comme langage vernaculaire de la contestation: Tunisie 2008-2014, thèse de doctorat, Universisté Paris 8, 2016.

Ricœur (Paul), « L'identité narrative », Esprit, nº 7-8, 1988, pp. 295-304.

Segrestin (Denis), « Les communautés pertinentes de l'action collective : canevas pour l'étude des fondements sociaux et des conflits du travail en France », Revue française de sociologie, vol. 21, $\mathrm{n}^{\circ} 2$, 1980, pp. 171-202.

Shirky (Clay), Cognitive Surplus: Creativity and Generosity in a Connected Age, Londres, Penguin Press, 2011. 
Shirky (Clay), Here Comes Everybody. The Power of Organizing without Organizations, Londres, Penguin Books, 2008.

Suarez Collado (Angela), « Mouvements sociaux sur la Toile Numérique : les effets des TIC sur le militantisme amazigh au Maroc », in Najar (Sihem) dir., Le cyberactivisme au Maghreb et dans le monde arabe, Tunis/Paris, IRMC/Karthala, 2013, pp. 41-54.

Taylor (Verta), Whittier (Nancy E.), « Collective identity in social movement communities: Lesbian movement mobilization», in Morris (Aldon), Mueller (Caroll) eds., Frontiers in social movements theory, New Haven, Yale University Press, 1992, pp. 104-129.

Tilly (Charles), From Mobilization to Revolution, Reading, Addison-Wesley, 1978.

Tilly (Charles), Tarrow (Sidney), Politique(s) du Conflit. De la grève à la révolution, Paris, Presses de SciencesPo, 2015.

Tonkin (Emma), Pfeiffer (Heather D.), Tourte (Gregory), « Twitter, information sharing and the London riots ", Bulletin of the American Society for Information Society and Technology, vol. 38, $\mathrm{n}^{\circ} 2$, 2012, pp. 49-57.

Traïni (Christophe), « Les émotions de la cause animale. Histoires affectives et travail militant », Politix, n 93, 2011, pp. 69-92.

Traïni (Christophe) dir., Émotions... Mobilisation!, Paris, Presses de SciencesPo, 2009, pp. 11-34.

Traïni (Christophe), Siméant (Johanna), « Introduction. Pourquoi et comment sensibiliser à la cause ?», in Traïni (Christophe) dir., Émotions... Mobilisation!, Paris, Presses de SciencesPo, 2009, pp. 11-34.

Turkle (Sherry), « Cyberspace and Identity », Contemporary Sociology, vol. 28, n 6, 1999, pp. 643-648.

\section{NOTES}

1. Cet article reprend quelques développement d'un ouvrage à paraître dans la collection "Sciences sociales» aux Presses de Mines (2017): Granjon (Fabien), avec la collaboration de Venetia Papa et Gökçe Tuncel, Mobilisations numériques. Politiques du conflit et technologies médiatiques.

2. Par l'emploi du syntagme " politiques du conflit », Charles Tilly et Sidney Tarrow entendent "prendre en considération une gamme de luttes politiques beaucoup plus large que celle habituellement prise en compte par la littérature consacrée aux mouvements sociaux ": Tilly (Charles), Tarrow (Sidney), Politique(s) du Conflit. De la grève à la révolution, Paris, Presses de SciencesPo, 2015, p. 14. Et d'ajouter: "La politique du conflit est faite d'interactions où des acteurs élèvent des revendications touchant aux intérêts d'autres acteurs, ce qui conduit à la coordination des efforts au nom d'intérêts ou de programmes partagés; et où l'État se trouve impliqué, soit en tant que destinataire de la revendication, soit comme instigateur, soit comme tierce partie. La politique du conflit réunit donc trois éléments bien connus de la vie sociale : le conflit, l'action collective et la politique. Le conflit implique qu'on pose une exigence portant atteinte aux intérêts de quelqu'un d'autre. [...] L'action collective, c'est la coordination des efforts au nom d'intérêts ou de programmes partagés. [...] On entre dans la politique quand on a affaire à des représentants de l'État, soit directement, soit en se lançant dans une activité qui touche aux droits de l'État, à ses règlements ou à ses intérêts " : ibid., pp. 26-27 et 28.

3. Negt (Oskar), L'espace public oppositionnel, Paris, Payot, 2007. 
4. Granjon (Fabien), L'Internet militant. Mouvement social et usages des réseaux télématiques, Rennes, Apogée, 2001.

5. Bert Klandermans montre qu'un trait essentiel de l'engagement dans l'action tient au fait que l'on soit persuadé que d'autres vont également s'engager: Klandermans (Bert), « Mobilization and Participation: Social-Psychological Expansions of Resource Mobilization Theory », American Sociological Review, vol. 49, $\mathrm{n}^{\circ}$ 5, 1984, pp. 583-600.

6. Oberschall (Anthony), Social Conflict and Social Movements, Englewood Cliffs, Prentice Hall, 1973.

7. Segrestin (Denis), «Les communautés pertinentes de l'action collective : canevas pour l'étude des fondements sociaux et des conflits du travail en France ", Revue française de sociologie, vol. 21, $\mathrm{n}^{\circ}$ 2, 1980, pp. 171-202.

8. Shirky (Clay), Cognitive Surplus: Creativity and Generosity in a Connected Age, Londres, Penguin Press, 2011.

9. Badouard (Romain), « Les mobilisations de clavier. Le lien hypertexte comme ressource des actions collectives en ligne », Réseaux, vol. 5, n 181, 2013, p. 93.

10. Khasnabish (Alex), «Insurgent Imaginations », Ephemera, vol. 7, n 4, 2007, pp. 505-525.

11. Pleyers (Geoffrey), Glasius (Marlies), «La résonnance des "mouvements des places": connexions, émotions, valeurs ", Socio, $n^{\circ} 2,2013$, p. 61.

12. Tilly (Charles), From Mobilization to Revolution, Reading, Addison-Wesley, 1978.

13. McAdam (Doug), Freedom Summer, Oxford, Oxford University Press, 1988; McAdam (Doug), Political Process and the Development of Black Insurgency (1930-1970), Chicago, The University of Chicago Press, 1982.

14. Bauman (Zygmunt), The Individualized Society, Cambridge, Polity Press, 2001 ; Poster (Mark), Information Please: Culture and Politics in the Age of Digital Machines, Durham, Duke University Press, 2006.

15. Granjon (Fabien), Lelong (Benoît), «Capital social, stratifications et technologies de l'information et de la communication. Une revue des travaux français et anglo-saxons ", Réseaux, vol. 24, $\mathrm{n}^{\circ} 139,2006$, pp. 147-182.

16. Nunes (Rodriguo), Organisation of the organisationless: collective action after networks, Lüneburg, Mute Books/PML Books, 2014, p. 24.

17. Mason (Paul), Why It's Kicking Off Everywhere: The New Global Revolutions, Londres, Verso, 2012.

18. Gerbaudo (Paolo), Tweets and the Streets: Social Media and Contemporary Activism, London, Pluto Press, 2012, p. 150.

19. Ibid.

20. Juris (Jeffrey), «Reflections on \#Occupy Everywhere: Social Media, Public Space, and Emerging Logics of Aggregation », American Ethnologist, vol. 39, n² 2, 2012, pp. 259-279.

21. Gerbaudo (Paolo), op. cit., p. 36.

22. Juris (Jeffrey), Networking futures: The movements against corporate globalization, Durham, Duke University Press, 2008.

23. L'usage de Mumble a par exemple permis que se tiennent des réunions (Transnational Mumbles Forum) rassemblant différents protagonistes des mouvements de crise de plusieurs pays et continents.

24. Fuchs (Christian), OccupyMedia! The Occupy Movement and Social Media in Crisis Capitalism, Winchester, Zero Books, 2013.

25. Gerbaudo (Paolo), op. cit., pp. 154-155.

26. Bruns (Axel), Highfield (Tim), « The Arab Spring on Twitter: Language Communities in \#egypt an \#lybia ", in Bebawi (Saba), Bossio (Diana) eds., Social Media and the Politics of reportage. The «Arab Spring ", New York, Palgrave Macmillan, 2014, p. 33.

27. Gerbaudo (Paolo), op. cit., p. 40. 
28. Bennett (Lance), Segerberg (Alexandra), The Logic of Connective Action. Digital Media and the Personalization of Contentious Politics, New York, Cambridge University Press, 2014 ; Bennett (Lance), Segerberg (Alexandra), «The logic of connective action: Digital media and the personalization of contentious politics ", Information, Communication \& Society, vol. 15, $\mathrm{n}^{\circ} 5,2012$, pp. 739-768.

29. Pour Bennett et Segerberg, les logiques de l'action collective relèvent de logiques d'agrégation d'actions politiques personnelles. Nous serions ainsi les témoins d'une évolution majeure : la «personnalisation des causes » dont la «collectivisation » serait, pour l'essentiel, rendue possible par l'usage des médias numériques. Alors que la politique conventionnelle serait largement fragilisée, le développement des «issue politics " visant à mobiliser les individus autour de leurs préoccupations personnelles serait en pleine expansion, notamment au travers de la constitution de communautés en ligne.

30. Stefania Milan évoque, pour sa part, l'existence d'un "cloud of protesting ", afin de rendre compte de la manière dont les SNS permettent que se déploie une forme d'action collective de plus en plus « désincarnée, distribuée et individualisée »: Milan (Stefania), Social movements and their technologies. Wiring social change, New York, Palgrave Macmillan, 2013.

31. Cristancho (Camilo), Anduiza (Eva), "Connective Action in European Mass Protest», communication aux ECPR Joint Sessions of Workshops Mainz, 11-16 March 2013, http:// www.lapietradialogues.org/area/pubblicazioni/doc000075.pdf.

32. Jenkins (Henry), "Cultural Acupuncture: Fan Activism and the Harry Potter Alliance», Transformative Works and Cultures, $\mathrm{n}^{\circ}$ 10, 2012, http://journal.transformativeworks.org/ index.php/twc/article/view/305/259.

33. Castells (Manuel), Networks of Outrage and Hope. Social movements in the Internet Age, Cambridge/ Malden, Polity Press, 2012.

34. Mathieu (Lilian), Comment lutter ? Sociologie et mouvements sociaux, Paris, Textuel, 2004.

35. Castells (Manuel), op. cit., pp. 144-145.

36. Ibid., p. 234.

37. Shirky (Clay), Here Comes Everybody. The Power of Organizing without Organizations, Londres, Penguin Books, 2008.

38. Castells (Manuel), op. cit., p. 139.

39. Benski (Tova), Langman (Lauren), "The effects of affects: the place of emotions in the mobilizations of $2011 »$, Current Sociology, vol. 61, n 4, 2013, pp. 525-540.

40. Goodwin (Jeff), Jasper (James M.), Polletta (Francesca) eds., Passionate Politics. Emotions and Social Movement, Chicago, University of Chicago Press, 2001.

41. Jasper (James M.), «Emotions and Social Movements: Twenty Years of Theory and Research », Annual Review of Sociology, $\mathrm{n}^{\circ}$ 37, 2011, pp. 285-303.

42. Stéphane Latté, montre également l'importance des expressions publiques d'émotions au sein du répertoire d'action victimaire: Latté (Stéphane), «Des "mouvements émotionnels" à la mobilisation des émotions ", Terrains/Théories, $n^{\circ} 2$, 2015, http://teth.revues.org/244. Cf. Traïni (Christophe), « Les émotions de la cause animale. Histoires affectives et travail militant », Politix, $\mathrm{n}^{\circ}$ 93, 2011, pp. 69-92; Traïni (Christophe) dir., Émotions... Mobilisation !, Paris, Presses de SciencesPo, 2009, pp. 11-34 ; Traïni (Christophe), Siméant (Johanna), « Introduction. Pourquoi et comment sensibiliser à la cause ? ", in Traïni (Christophe) dir., Émotions... Mobilisation !, Paris, Presses de SciencesPo, 2009, pp. 11-34.

43. Jasper (James M.), The Art of Moral Protest. Culture, Biography, and Creativity in Social Movements, Chicago, University of Chicago Press, 1997.

44. Gerbaudo (Paolo), op. cit., p. 12.

45. Tonkin (Emma), Pfeiffer (Heather D.), Tourte (Gregory), « Twitter, information sharing and the London riots ", Bulletin of the American Society for Information Society and Technology, vol. 38, $\mathrm{n}^{\circ}$ 2, 2012, pp. 49-57. 
46. Gerbaudo (Paolo), op. cit., p. 87.

47. Ibid., p. 159 sq.

48. Mitrović (Marjira), Paltoglou (Georgios), Tadić (Bosiljka), « Quantitative analysis of bloggers' collective behavior powered by emotions ", Journal of Statistical Mechanics: Theory and Experiment, $\mathrm{n}^{\circ}$ 2, 2011, http://www.scl.rs/papers/Mitrovic_JSTAT_2011.pdf.

49. Badouard (Romain), «"Je ne suis pas Charlie" : pluralité des prises de paroles sur le web et les réseaux sociaux", in Lefebure (Pierre), Secail (Claire), Le défi Charlie, Les médias à l'épreuve des attentants, Paris, Le Mieux-Éditeur, 2015, pp. 196.

50. Nunes (Rodriguo), Organisation of the organisationless: collective action after networks, Lüneburg, Mute Books/PML Books, 2014; Alhassen (Maytha), Shihab-Eldin (Ahmed) eds., Demanding Dignity: Young Voices from the Arab Spring, Ashland, White Cloud, 2012.

51. Gerbaudo (Paolo), op. cit., p. 14.

52. Ghonim (Wael), Révolution 2.0. Le pouvoir des gens plus fort que les gens au pouvoir, Paris, Steinkis, 2012, p. 88.

53. Riboni (Ulrike L.), «Juste un peu de vidéo ». La vidéo partagée comme langage vernaculaire de la contestation : Tunisie 2008-2014, thèse de doctorat, Universisté Paris 8, 2016, p. 152.

54. Boëx (Cécile), «La vidéo comme outil de l'action collective et de la lutte armée », in Burgat (Françoise), Paoli (Bruno), Pas de printemps pour la Syrie. Les clés pour comprendre les acteurs et les défis de la crise [2011-2013], Paris, La Découverte, 2013, p.175.

55. Contestant et déplaçant les règles du jeu politique en y introduisant des nouvelles revendications sociales et culturelles, Alberto Melucci estime que les NMS sont le fait d'individus guidés par la volonté « de revendiquer collectivement le droit de réaliser leur propre identité ; le droit de disposer de leur créativité personnelle, de leur vie affective et de leur existence biologique et interpersonnelle » : Melucci (Alberto), « The New Social Movements: A Theoretical Approach ", Social Science Information, $n^{\circ} 19,1980$, p. 218.

56. Toutefois, les principales différences entre les "nouveaux» mouvements sociaux et les formes plus classiques d'action collective ne résident pas seulement dans leur base sociale, leurs objectifs et leurs exigences, mais aussi dans leur capacité à créer un nouvel espace politique bousculant la distinction traditionnelle établie entre État et société civile, un espace public intermédiaire dont la fonction est de rendre visibles des problèmes publics qui peuvent faire l'objet d'une traduction politique, notamment en termes de droits. Aussi, l'usage du syntagme «identity politics » permet de désigner les logiques d'engagement qui valorisent « l'expérience propre du sujet " au sein des luttes qu'il mène et la construction identitaire d'apparaitre alors comme un ressort essentiel de l'action collective: Offe (Claus), " New social movements: Challenging the boundaries of institutional politics ", Social Research, vol. 52, n 4, 1985, pp. 817868 ; Melucci (Alberto), « The symbolic challenge of contemporary movements ", Social research, vol. 52, n 4, 1985, pp. 789-816.

57. Papa (Venetia), Milioni (Dimitra), « I don't wear blinkers alright? An analysis of civic identity in the Indignados movement and the role of Facebook ", Javnost-The Public, vol. 23, n 3, 2016, pp. 290-306.

58. McAdam (Doug), Freedom Summer, op. cit.

59. Melucci (Alberto), « Mouvements sociaux, mouvements post-politiques », Revue internationale d'action communautaire, $\mathrm{n}^{\circ} 10,1983$, pp. 13-30.

60. Gaxie (Daniel), «Économie des partis et rétributions du militantisme », Revue française de science politique, vol. 27, $\mathrm{n}^{\circ} 1,1977$, pp. 123-154.

61. Suarez Collado (Angela), « Mouvements sociaux sur la Toile Numérique : les effets des TIC sur le militantisme amazigh au Maroc ", in Najar (Sihem) dir., Le cyberactivisme au Maghreb et dans le monde arabe, Tunis/Paris, IRMC/Karthala, 2013, pp. 41-54. 
62. Taylor (Verta), Whittier (Nancy E.), « Collective identity in social movement communities: Lesbian movement mobilization», in Morris (Aldon), Mueller (Caroll) eds., Frontiers in social movements theory , New Haven, Yale University Press, 1992, pp. 104-129.

63. Granjon (Fabien), «Attac-info: entre communauté d'action et espace de représentation. Ethnographie d'un média "alter" lors du Forum social mondial 2003 ", Matériaux pour l'histoire de notre temps, $\mathrm{n}^{\circ}$ 79, 2005, pp. 70-76.

64. Melucci, art. cit., p. 15.

65. Ibid.

66. Telle que définie par Axel Honneth, la logique du rapport de reconnaissance relève d'une confirmation des dimensions « identitaires » des sujets concourant à leur réalisation individuelle. Sans rentrer dans le détail du dispositif conceptuel du philosophe allemand, précisons que, pour lui, l'une des dimensions essentielles de la reconnaissance repose sur le principe d'un sujet qui se conçoit réflexivement comme appartenant à une société organisée. Ce faisant, la reconnaissance est liée à une forme de relation pratique à soi-même assurant la valeur sociale de son identité et ouvrant à l'estime sociale de soi, c'est-à-dire à «l'attitude positive qu'un individu est capable d'adopter à l'égard de lui-même lorsqu'il est reconnu par les membres de sa communauté comme une personne d'un certain genre »: Honneth (Axel), La lutte pour la reconnaissance, Paris, Cerf, 2000, p. 97. Pour Honneth, cette voie dans la reconnaissance habilite l'individu à se rapporter positivement à ses qualités personnelles et singulières en tant qu'il s'appuie sur des fins éthiques qui imprègnent la société à laquelle il participe.

67. Turkle (Sherry), « Cyberspace and Identity », Contemporary Sociology, vol. 28, n 6, 1999, pp. 643-648.

68. Castells (Manuel), op. cit., pp. 144-145.

69. Denouël (Julie), Granjon (Fabien), Aubert (Aurélie), Médias numériques et participation. Entre engagement citoyen et production de soi, Paris, Mare \& Martin, 2014.

70. Ricœur (Paul), «L'identité narrative », Esprit, n 7-8, 1988, pp. 295-304.

71. Pleyers (Geoffrey), Capitaine (Brieg) dir., Mouvements sociaux. Quand le sujet devient acteur, Paris, Éditions de la Maison des sciences de l'homme, 2016 , p. 8.

72. Lecomte (Romain), «Les usages "citoyens" d'Internet dans le contexte autoritaire tunisien: analyse de l'émergence d'un nouvel espace public de la critique ", in Najar (Sihem) dir., Le cyberactivisme au Maghreb et dans le monde arabe, Tunis/Paris, IRMC/Karthala, 2013, p. 69.

73. Ibid.

74. Chadwick (Peter), Continuum mechanics: concise theory and problems, Londres, Courier Corporation, 2012.

75. Dahlgren (Peter), « Reconfigurer la culture civique dans un milieu médiatique en évolution », Questions de communication, $\mathrm{n}^{\circ}$ 3, 2003, pp. 151-168.

76. Lecomte (Romain), art. cit., p. 71.

77. Khosrokhavar (Farhad), The New Arab Revolutions that Shook the World, Boulder, Paradigm Publishers, 2012.

78. Dahlgren (Peter), The political web: Media, participation and alternative democracy, Londres, Palgrave Macmillan, 2013; Dahlgren (Peter), « The Internet, public spheres, and political communication: dispersion and deliberation ", Political Communication, $\mathrm{n}^{\circ}$ 22, 2005, pp. 147-162. 79. Prentoulis (Marina), Thomassen, (Lasse), «Political theory in the square: Protest, representation and subjectification ", Contemporary Political Theory, vol. 12, n 3, 2013, pp. 166184.

80. Ion (Jacques) dir., L'engagement au pluriel, Saint-Étienne, Publications de l'Université de Saint-Étienne, 2001, p. 29.

81. Klandermans (Bert), «Transient Identities? Membership Patterns in the Dutch Peace Movement ", in Laraña (Enrique), Johnston (Hank), Gusfield (Joseph R.), eds., New Social Movement. From Ideology to Identity, Philadelphie, Temple University Press, 1994, pp. 168-184. 
82. L'appui de certains médias peut néanmoins, parfois conférer une notoriété élargie aux actions et à l'identité collective des mouvements et permettre de consolider les représentations et les cadrages élaborés au cœur des dispositifs en ligne.

83. Fenton (Natalie), Barassi (Veronica), « Alternative media and social networking sites: The politics of individuation and political participation ", The Communication Review, vol. 14, $\mathrm{n}^{\circ} 3$, 2011, pp. 179-196.

84. Il faut ici signaler que, si la multiplication des flux d'information est un marqueur de l'époque, la possibilité d'initier des interactions inédites via l'usage des TNIC ne relève toutefois en rien d'un automatisme tant cet usage reste soumis aux diverses formes de capitaux qui en dessinent nécessairement les contours (Urry, 2007), y compris dans l'espace des mouvements sociaux.

85. Halleck (DeeDee), Hand-held Visions. The Impossible Possibilities of Community Media, New York, Fordham University Press, 2002.

86. Bimber (Bruce), Flanagin (Andrew J.), Stohl (Cynthia), « Reconceptualizing Collective Action in the Contemporary Media Environment ", Communication Theory, vol. 15, n 4, 2005, pp 365-388.

87. Dans les années 1990, la sociologie des usages des TIC va s'appuyer sur le concept de (double) médiation, préconisant ainsi l'analyse concrète d'une articulation sociotechnique. Cette idée de la double médiation de la technique et du social va constituer un principe pour de nombreuses recherches, permettant de «sortir d'une instrumentalisation de la technique qui évacue les enjeux sociaux dont celle-ci est investie, [mais aussi] de réfuter le schéma réducteur du déterminisme social qui fait l'impasse sur la technique et voit, a contrario, le social comme un ensemble d'acteurs autonomes qui donneraient forme à une technique à la plasticité infinie »: Chambat (Pierre), Jouët (Josiane), "Machines à communiquer: acquis et interrogation», in dixième Congrès National des SIC, Grenoble-Echirolles, SFSIC, 1996, p. 211.

\section{INDEX}

Mots-clés : critique sociale, internet, mobilisation de l'action, mouvements sociaux, TNIC

\section{AUTEUR}

\section{FABIEN GRANJON}

Fabien Granjon est sociologue, professeur en sciences de l'information et de la communication et directeur du Centre d'Études sur les Médias, les Technologies et l'Internationalisation (CEMTI) de l'Université Paris 8 Vincennes à Saint-Denis. 